Proyecciones Journal of Mathematics

Vol. 37, No 3, pp. 535-546, September 2018.

Universidad Católica del Norte

Antofagasta - Chile

\title{
On some double sequence spaces of interval number
}

\author{
Sibel Yasemin Gölbol \\ Mersin University, Turkey \\ Ayhan Esi \\ Adiyaman University, Turkey \\ and

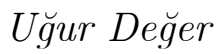 \\ Mersin University, Turkey \\ Received: October 2017. Accepted : November 2017
}

\begin{abstract}
Esi and Yasemin [9] defined the metric spaces $\bar{c}_{0}(f, p, s), \bar{c}(f, p, s)$, $\bar{l}_{\infty}(f, p, s)$ and $\bar{l}_{p}(f, p, s)$ of sequences of interval numbers by a modulus function. In this study, we consider a generalization for double sequences of these metric spaces by taking a $\psi$ function, satisfying the following conditions, instead of s parameter. For this aim, let $\psi(k, l)$ be a positive function for all $k, l \in \mathbf{N}$ such that

(i) $\lim _{k, l \rightarrow \infty} \psi(k, l)=0$,

(ii) $\Delta_{2} \psi(k, l)=\psi(k-1, l-1)-2 \psi(k, l)+\psi(k+1, l+1) \geq 0$.

or

$$
\psi(k, l)=1 .
$$

Therefore, according to class of functions which satisfying the conditions $(i)$ and (ii) we deal with the metric spaces $\bar{c}_{0}^{2}(f, p, \psi), \bar{c}^{2}(f, p, \psi)$, $\bar{l}_{\infty}^{2}(f, p, \psi)$ and $\bar{l}_{p}^{2}(f, p, \psi)$ of double sequences of interval numbers defined by a modulus function.
\end{abstract}

Subjclass [2010] : 46A45, 54E50, 40A05, 40C05, 40J05.

Keywords : Complete space, interval number, modulus function, double sequence space. 


\section{Introduction}

The concept of interval arithmetic was first introduced by Dwyer in [2]. Interval arithmetic or interval analysis is a method developed by mathematicians since the 1950s and 1960s as an approach to putting bounds on matrix computation errors, rounding errors and measurement errors in mathematical computation and thus developing numerical methods that yield reliable results, [2], [3], [17], [20], [21]. One approach to dealing with such numerical errors is a technique called interval arithmetic. In 2002, Kuo-Ping Chiao gave the sequences of interval numbers and defined usual convergence of sequences of interval number. In subsequent years, M. Şengönül and A. Eryılmaz studied bounded and convergent sequence spaces of interval numbers in [24] and proved that these spaces are complete metric space. In [4]-[8],[9],[13],[14],[15] and [16] studied different properties of interval numbers.

Let us recall some definitions related to interval numbers. The set of all real valued closed intervals is denoted by $\mathbf{R}$. Any element of $\mathbf{R}$ is called interval number and denoted by $\bar{A}=\left[x_{l}, x_{r}\right]$. Assume that $x_{l}$ and $x_{r}$ are first and last points of $\bar{A}$ interval number, respectively. Then for $\bar{A}_{1}, \bar{A}_{2} \in \mathbf{R}$

1. $\bar{A}_{1}=\bar{A}_{2} \Leftrightarrow x_{1_{l}}=x_{2_{l}}, x_{1_{r}}=x_{2_{r}}$

2. $\bar{A}_{1}+\bar{A}_{2}=\left\{x \in \mathbf{R}: x_{1_{l}}+x_{2_{l}} \leq x \leq x_{1_{r}}+x_{2_{r}}\right\}$

3. $\alpha \bar{A}_{1}= \begin{cases}\left\{x \in \mathbf{R}: \alpha x_{1_{r}} \leq x \leq \alpha x_{1_{l}}\right\}, & \alpha<0 ; \\ \left\{x \in \mathbf{R}: \alpha x_{1_{l}} \leq x \leq \alpha x_{1_{r}}\right\}, & \alpha \geq 0 .\end{cases}$

4. $\bar{A}_{1} \cdot \bar{A}_{2}=\left\{x \in \mathbf{R}: \min \left\{x_{1_{i}} \cdot x_{2_{j}} ; i, j=l, r\right\} \leq x \leq \max \left\{x_{1_{i}} \cdot x_{2_{j}} ; i, j=l, r\right\}\right\}$. In [22], we know that the set $\mathbf{R}$ is a complete metric space by the metric

$\bar{d}\left(\bar{A}_{1}, \bar{A}_{2}\right)=\max \left\{\left|x_{1_{l}}-x_{2_{l}}\right|,\left|x_{1_{r}}-x_{2_{r}}\right|\right\}$.

In case $\bar{A}_{1}=[a, a]$ and $\bar{A}_{2}=[b, b]$, it coincides with usual metric of $\mathbf{R}$. The sequence $(f(k))=\bar{A}=\left(\bar{A}_{k}\right)$ defined with transformation $f: \mathbf{N} \rightarrow \mathbf{R}$ is called sequence of interval numbers and the $\bar{A}_{k}$ is called $k^{\text {th }}$ term of sequence $\bar{A}=\left(\bar{A}_{k}\right)$. $\bar{\omega}$ denotes the set of all sequences of interval numbers with real terms and the algebraic properties of $\bar{\omega}$ can be found in [19].

The definition of convergence of sequences of interval numbers as follows [1]:

A sequence $\bar{A}=\left(\bar{A}_{k}\right)$ of interval numbers is said to be convergent to the interval number $\bar{A}_{0}$ if for each $\epsilon>0$ there exists a positive integer $k_{0}$ such that $\bar{d}\left(\bar{A}_{k}, \bar{A}_{0}\right)<\epsilon$ for all $k \geq k_{0}$ and we denote it by $\lim _{k} \bar{A}_{k}=\bar{A}_{0}$. Thus, 


$$
\lim _{k} \bar{A}_{k}=\bar{A}_{0} \Leftrightarrow \lim _{k} A_{k_{l}}=A_{0_{l}}, \lim _{k} A_{k_{r}}=A_{0_{r}} .
$$

We recall that modulus function is a function $f:[0, \infty) \rightarrow[0, \infty)$ such that $(a) f(x)=0$ if and only if $x=0,(b) f(x+y) \leq f(x)+f(y)$ for all $x, y \geq 0,(c) f$ is increasing, $(d) f$ is continuous from the right at zero.

It follows from $(a)$ and $(d)$ that $f$ must be continuous everywhere on $[0, \infty)$.

Let $p=\left(p_{k}\right)$ be a bounded sequence of strictly positive real numbers. If $H=\sup _{k} p_{k}$, then for any complex numbers $a_{k}$ and $b_{k}$

$$
\left|a_{k}+b_{k}\right|^{p_{k}} \leq C\left(\left|a_{k}\right|^{p_{k}}+\left|b_{k}\right|^{p_{k}}\right),
$$

where $C=\max \left(1,2^{H-1}\right)$.

An interval sequence $\bar{x}=\left(\bar{x}_{k}\right) \in \bar{\omega}$ is said to be interval Cauchy sequence if for every $\epsilon>0$ there exists a $k_{0} \in \mathbf{N}$ such that $\bar{d}\left(\bar{x}_{n}, \bar{x}_{m}\right)<\epsilon$ whenever $n, m>k_{0}$.

Now we give the definition of solid:

A set of $X$ sequences of interval numbers is said to be solid (or normal) if $\left(\bar{B}_{k}\right) \in X$ whenever $\bar{d}\left(\bar{B}_{k}, \overline{0}\right) \leq \bar{d}\left(\bar{A}_{k}, \overline{0}\right)$ for all $k \in \mathbf{N}$, for some $\left(\bar{A}_{k}\right) \in X$.

\section{Preliminaries}

Let us define transformation $f$ from $\mathbf{N} \times \mathbf{N}$ to $\mathbf{R}$ by $f(i, j)=\left(\bar{x}_{i, j}\right)$. Then $\bar{x}=\left(\bar{x}_{i, j}\right)$ is called sequence of double interval numbers. The $\bar{x}_{i, j}$ is called $(i, j)$ th term of sequence $\bar{x}[12]$.

An interval valued double sequence $\left(\bar{A}_{k, l}\right)$ is said to be convergent in the Pringsheim sense to an interval number $\bar{A}_{0}$ if for every $\epsilon>0$ there exists a $n \in \mathbf{N}$ such that

$$
\bar{d}\left(\bar{A}_{k, l}, \bar{A}_{0}\right)<\epsilon
$$

for $k, l \geq n$. In this case we write $P-\lim \bar{A}_{k, l}=\bar{A}_{0}$ [23].

We denote by $\bar{c}^{2}$ the set of all convergent in the Pringsheim sense double sequence of interval numbers.

An interval valued double sequence $\left(\bar{A}_{k, l}\right)$ is said to be bounded if there exists a positive number $B$ such that $\bar{d}\left(\bar{A}_{k, l}, \overline{0}\right) \leq B$ for all $k, l \in \mathbf{N}$.

We will denote the set of all bounded double sequences of interval numbers by the symbol $\bar{l}_{\infty}^{2}$. It should be noted that $\bar{c}^{2}$ is not the subset of $\bar{l}_{\infty}^{2}$ 
The definition of solid for double sequence spaces of interval numbers is given as follows:

An interval valued double sequence spaces $\bar{E}^{2}$ is said to be solid if $\bar{y}=\left(\bar{y}_{k, l}\right) \in \bar{E}^{2}$ whenever $\left|\bar{y}_{k, l}\right| \leq\left|\bar{x}_{k, l}\right|$ for all $k, l \in \mathbf{N}$ and $\bar{x}=\left(\bar{x}_{k, l}\right) \in$ $\bar{E}^{2}[10]$.

Let $p=\left(p_{i, j}\right)$ be a double sequence of positive real numbers. If $0<$ $p_{i, j} \leq \sup _{i, j} p_{i, j}=H<\infty$ and $D=\max \left(1,2^{H-1}\right)$, then for $a_{i, j}, b_{i, j}$ for all $i, j \in \mathbf{N}$, we have

$$
\left|a_{i, j}+b_{i, j}\right|^{p_{i, j}} \leq D\left(\left|a_{i, j}\right|^{p_{i, j}}+\left|b_{i, j}\right|^{p_{i, j}}\right) .
$$

On the other hand $\psi(k, l)$ be a positive function for all $k, l \in \mathbf{N}$ such that

$\lim _{k, l \rightarrow \infty} \psi(k, l)=0$,

$$
\Delta_{2} \psi(k, l)=\psi(k-1, l-1)-2 \psi(k, l)+\psi(k+1, l+1) \geq 0
$$

or

$\psi(k, l)=1$.

We will show that $M$ is the set of all $\psi(k, l)$ that provide above conditions.

Suppose that $f$ is a modulus function, $p=\left(p_{k, l}\right)$ is a sequence of strictly positive real numbers and $\psi \in M$. Accordingly, the sets of interval number sequences which generalize to the sets of double sequences of interval numbers introduced in [9] are given as follows.

$$
\begin{aligned}
& \bar{c}_{0}^{2}(f, p, \psi)=\left\{\bar{A}=\left(\bar{A}_{k, l}\right): \lim _{k, l} \psi(k, l)\left[f\left(\bar{d}\left(\bar{A}_{k, l}, \overline{0}\right)\right)\right]^{p_{k, l}}=0, \psi \in M\right\} \\
& \bar{c}^{2}(f, p, \psi)=\left\{\bar{A}=\left(\bar{A}_{k, l}\right): \lim _{k, l} \psi(k, l)\left[f\left(\bar{d}\left(\bar{A}_{k, l}, \bar{A}_{0}\right)\right)\right]^{p_{k, l}}=0, \psi \in M\right\} \\
& \bar{l}_{\infty}^{2}(f, p, \psi)=\left\{\bar{A}=\left(\bar{A}_{k, l}\right): \sup _{k, l} \psi(k, l)\left[f\left(\bar{d}\left(\bar{A}_{k, l}, \overline{0}\right)\right)\right]^{p_{k, l}}<\infty, \psi \in M\right\}
\end{aligned}
$$
and

$\bar{l}_{p}^{2}(f, p, \psi)=\left\{\bar{A}=\left(\bar{A}_{k, l}\right): \sum_{k, l} \psi(k, l)\left[f\left(\bar{d}\left(\bar{A}_{k, l}, \overline{0}\right)\right)\right]^{p_{k, l}}<\infty, \psi \in M\right\}$.

Aim of this study is to give the metric spaces $\bar{c}_{0}^{2}(f, p, \psi), \bar{c}^{2}(f, p, \psi)$, $\bar{l}_{\infty}^{2}(f, p, \psi)$ and $\bar{l}_{p}^{2}(f, p, \psi)$ of double sequences of interval numbers and investigate the relations between them.

\section{Main results}

Now, we may begin with the following theorem. 
The sets $\bar{c}_{0}^{2}(f, p, \psi), \bar{c}^{2}(f, p, \psi), \bar{l}_{\infty}^{2}(f, p, \psi)$ and $\bar{l}_{p}^{2}(f, p, \psi)$ of double sequences of interval numbers are closed under the coordinate wise addition and scalar multiplication.

Proof. We consider only the space $\bar{l}_{\infty}^{2}(f, p, \psi)$. Let us define

$+: \bar{l}_{\infty}^{2}(f, p, \psi) \times \bar{l}_{\infty}^{2}(f, p, \psi) \rightarrow \bar{l}_{\infty}^{2}(f, p, \psi)$

• : $\mathbf{R} \times \bar{l}_{\infty}^{2}(f, p, \psi) \rightarrow \bar{l}_{\infty}^{2}(f, p, \psi)$.

Suppose that $\bar{A}, \bar{B} \in \bar{l}_{\infty}^{2}(f, p, \psi)$. Then we can write $\sup _{k, l} \psi(k, l)\left[f\left(\bar{d}\left(\bar{A}_{k, l}, \overline{0}\right)\right)\right]^{p_{k, l}}<\infty$

and

$\sup _{k, l} \psi(k, l)\left[f\left(\bar{d}\left(\bar{B}_{k, l}, \overline{0}\right)\right)\right]^{p_{k, l}}<\infty$.

Since $\bar{d}\left(\bar{A}_{k, l}+\bar{B}_{k, l}, \overline{0}\right) \leq \bar{d}\left(\bar{A}_{k, l}, \overline{0}\right)+\bar{d}\left(\bar{B}_{k, l}, \overline{0}\right)$ and $f$ is a modulus function, then we have

$\mathrm{f}\left(\bar{d}\left(\bar{A}_{k, l}+\bar{B}_{k, l}, \overline{0}\right)\right) \leq f\left(\bar{d}\left(\bar{A}_{k, l}, \overline{0}\right)+\bar{d}\left(\bar{B}_{k, l}, \overline{0}\right)\right) \leq f\left(\bar{d}\left(\bar{A}_{k, l}, \overline{0}\right)\right)+f\left(\bar{d}\left(\bar{B}_{k, l}, \overline{0}\right)\right)$.

Also, since $p=\left(p_{k, l}\right)$ is a sequence of strictly positive real numbers, we can write $0 \leq h=\inf _{k, l} p_{k, l} \leq p_{k, l} \leq \sup _{k, l} p_{k, l}=H<\infty$. If we choose $M=\max \left(1,2^{H-1}\right)$, we get

$\left[\mathrm{f}\left(\bar{d}\left(\bar{A}_{k, l}+\bar{B}_{k, l}, \overline{0}\right)\right)\right]^{p_{k, l}} \leq\left[f\left(\bar{d}\left(\bar{A}_{k, l}, \overline{0}\right)\right)+f\left(\bar{d}\left(\bar{B}_{k, l}, \overline{0}\right)\right)\right]^{p_{k, l}}$

$\leq M\left[f\left(\bar{d}\left(\bar{A}_{k, l}, \overline{0}\right)\right)\right]^{p_{k, l}}+M\left[f\left(\bar{d}\left(\bar{B}_{k, l}, \overline{0}\right)\right)\right]^{p_{k, l}}$.

Because $\psi(k, l)$ is bounded, we obtain

$\sup _{k, l} \psi(k, l)\left[f\left(\bar{d}\left(\bar{A}_{k, l}+\bar{B}_{k, l}, \overline{0}\right)\right)\right]^{p_{k, l}}$

$\leq \sup _{k, l} \psi(k, l) M\left[f\left(\bar{d}\left(\bar{A}_{k, l}, \overline{0}\right)\right)\right]^{p_{k, l}}+\sup _{k, l} \psi(k, l) M\left[f\left(\bar{d}\left(\bar{B}_{k, l}, \overline{0}\right)\right)\right]^{p_{k, l}}<$ $\infty$.

Thus, $\bar{A}+\bar{B} \in \bar{l}_{\infty}^{2}(f, p, \psi)$.

Now, let $\bar{A} \in \bar{l}_{\infty}^{2}(f, p, \psi)$ and $\alpha \in \mathbf{R}$. Then

$\sup _{k, l} \psi(k, l)\left[f\left(\bar{d}\left(\bar{A}_{k, l}, \overline{0}\right)\right)\right]^{p_{k, l}}<\infty$.

Since $\bar{d}\left(\alpha \bar{A}_{k, l}, \overline{0}\right)=|\alpha| \bar{d}\left(\bar{A}_{k, l}, \overline{0}\right)$ and $\mathrm{f}$ is a modulus function, we can write

$\mathrm{f}\left(\bar{d}\left(\alpha \bar{A}_{k, l}, \overline{0}\right)\right)=f\left(|\alpha| \bar{d}\left(\bar{A}_{k, l}, \overline{0}\right)\right) \leq|\alpha| f\left(\bar{d}\left(\bar{A}_{k, l}, \overline{0}\right)\right)$.

Because $p=\left(p_{k, l}\right)$ is a sequence of strictly positive real numbers, we have

$\left[\mathrm{f}\left(\bar{d}\left(\alpha \bar{A}_{k, l}, \overline{0}\right)\right)\right]^{p_{k, l}} \leq|\alpha|^{p_{k, l}}\left[f\left(\bar{d}\left(\bar{A}_{k, l}, \overline{0}\right)\right)\right]^{p_{k, l}}$

and because $\psi(k, l)$ is bounded, we obtain

$\sup _{k, l} \psi(k, l)\left[f\left(\bar{d}\left(\alpha \bar{A}_{k, l}, \overline{0}\right)\right)\right]^{p_{k, l}} \leq \sup _{k, l} \psi(k, l)|\alpha|^{p_{k, l}}\left[f\left(\bar{d}\left(\bar{A}_{k, l}, \overline{0}\right)\right)\right]^{p_{k, l}}<$ $\infty$ 
Therefore, $\alpha \bar{A} \in \bar{l}_{\infty}^{2}(f, p, \psi)$.

The proof is similarly done for the spaces $\bar{c}_{0}^{2}(f, p, \psi), \bar{c}^{2}(f, p, \psi)$ and $\bar{l}_{p}^{2}(f, p, \psi)$.

The sets $\bar{c}_{0}^{2}(f, p, \psi), \bar{c}^{2}(f, p, \psi), \bar{l}_{\infty}^{2}(f, p, \psi)$ and $\bar{l}_{p}^{2}(f, p, \psi)$ of double sequences of interval numbers are complete metric spaces with respect to the metrics

$$
\bar{d}_{\infty}(\bar{A}, \bar{B})=\sup _{k, l} \psi(k, l)\left[f\left(\bar{d}\left(\bar{A}_{k, l}, \bar{B}_{k, l}\right)\right)\right]^{\frac{p_{k, l}}{M}}
$$

and

$$
\bar{d}_{p}(\bar{A}, \bar{B})=\left\{\sum_{k, l} \psi(k, l)\left[f\left(\bar{d}\left(\bar{A}_{k, l}, \bar{B}_{k, l}\right)\right)\right]^{p_{k, l}}\right\}^{\frac{1}{M}}
$$

respectively, where $\bar{A}=\left(\bar{A}_{k, l}\right)$ and $\bar{B}=\left(\bar{B}_{k, l}\right)$ are elements of the sets $\bar{c}_{0}^{2}(f, p, \psi), \bar{c}^{2}(f, p, \psi), \bar{l}_{\infty}^{2}(f, p, \psi)$ and $\bar{l}_{p}^{2}(f, p, \psi)$ and $M=\max \left(1, \sup _{k, l} p_{k, l}=\right.$ $H)$.

Proof. We consider only the space $\bar{c}_{0}^{2}(f, p, \psi)$, since the proof is similar for the spaces $\bar{c}^{2}(f, p, \psi), \bar{l}_{\infty}^{2}(f, p, \psi)$ and $\bar{l}_{p}^{2}(f, p, \psi)$. It is obviously seen that $\bar{d}_{\infty}$ is a metric on $\bar{c}_{0}^{2}(f, p, \psi)$. We will prove the completeness of the space $\bar{c}_{0}^{2}(f, p, \psi)$. Assume that $\left(\bar{A}^{i}\right)$ is any Cauchy sequence in the space $\bar{c}_{0}^{2}(f, p, \psi)$, where $\bar{A}^{i}=\left(\bar{A}_{k, 0}^{(i)}, \bar{A}_{k, 1}^{(i)}, \bar{A}_{k, 2}^{(i)}, \ldots\right)$ for all $k \in \mathbf{N}$. Therefore, for a given $\epsilon>0$ there exists a positive integer $n_{0}(\epsilon)$ such that

$$
\bar{d}_{\infty}\left(\bar{A}^{i}, \bar{A}^{j}\right)=\sup _{k, l} \psi(k, l)\left[f\left(\bar{d}\left(\bar{A}_{k, l}^{(i)}, \bar{A}_{k, l}^{(j)}\right)\right)\right]^{\frac{p_{k, l}}{M}}<\epsilon
$$

for all $i, j>n_{0}(\epsilon)$. We have for each fixed $k, l \in \mathbf{N}$ from (3.3) that

$$
\psi(k, l)\left[f\left(\bar{d}\left(\bar{A}_{k, l}^{(i)}, \bar{A}_{k, l}^{(j)}\right)\right)\right]^{\frac{p_{k, l}}{M}}<\epsilon
$$

for all $i, j>n_{0}(\epsilon)$. (3.4) means that

$$
\lim _{i, j \rightarrow \infty} \psi(k, l)\left[f\left(\bar{d}\left(\bar{A}_{k, l}^{(i)}, \bar{A}_{k, l}^{(j)}\right)\right)\right]^{\frac{p_{k, l}}{M}}=0 .
$$

Because $\psi(k, l) \neq 0$ for all $k, l \in \mathbf{N}$ and $f$ is continuous, we obtain from (3.5) that

$$
f\left[\lim _{i, j \rightarrow \infty}\left(\bar{d}\left(\bar{A}_{k, l}^{(i)}, \bar{A}_{k, l}^{(j)}\right)\right)\right]=0 .
$$


Thus, since $f$ is modulus function, we can get by (3.6) that

$$
\lim _{i, j \rightarrow \infty} \bar{d}\left(\bar{A}_{k, l}^{(i)}, \bar{A}_{k, l}^{(j)}\right)=0 .
$$

This means that $\left(\bar{A}_{k, l}^{(i)}\right)$ is a Cauchy sequence in $\mathbf{R}$ for every fixed $k, l \in \mathbf{N}$. Since $\mathbf{R}$ is complete, it converges, say $\bar{A}_{k, l}^{(i)} \rightarrow \bar{A}_{k, l}$ as $i \rightarrow \infty$. Using these infinitely many limits, we defined the interval sequence $\left(\bar{A}_{k, l}\right)=$ $\left(\bar{A}_{k, 0}, \bar{A}_{k, 1}, \bar{A}_{k, 2}, \ldots\right)$ for all $k \in \mathbf{N}$. Let us pass to limit firstly as $j \rightarrow \infty$ and nextly taking supremum over $k, l \in \mathbf{N}$ in (3.4) we have $\bar{d}_{\infty}\left(\bar{A}^{i}, \bar{A}_{k, l}\right) \leq \epsilon$. Since $\left(\bar{A}_{k, l}^{(i)}\right) \in \bar{c}_{0}^{2}(f, p, \psi)$ for each $i \in \mathbf{N}$, there exists a $k_{0} \in \mathbf{N}$ such that

$$
\psi(k, l)\left[f\left(\bar{d}\left(\bar{A}_{k, l}^{(i)}, \overline{0}\right)\right)\right]^{p_{k, l}}<\epsilon
$$

for every $k, l \geq k_{0}(\epsilon)$ and for each fixed $i \in \mathbf{N}$.

From triangle inequality, we can write

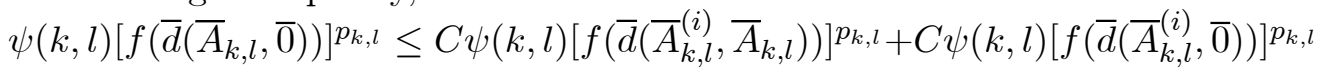

for all $i, k, l \in \mathbf{N}$, where $C=\max \left(1,2^{H-1}\right)$. Now for all $k, l \geq k_{0}(\epsilon)$, we obtain

$\psi(k, l)\left[f\left(\bar{d}\left(\bar{A}_{k, l}, \overline{0}\right)\right)\right]^{p_{k, l}} \leq 2 \epsilon$.

This gives that $\left(\bar{A}_{k, l}\right) \in \bar{c}_{0}^{2}(f, p, \psi)$. Because $\left(\bar{A}_{k, l}^{(i)}\right)$ was an arbitrary Cauchy sequence, the space $\bar{c}_{0}^{2}(f, p, \psi)$ is complete.

The spaces $\bar{c}_{0}^{2}(f, p, \psi), \bar{l}_{\infty}^{2}(f, p, \psi)$ and $\bar{l}_{p}^{2}(f, p, \psi)$ are solid.

Proof. Assume that $\bar{X}^{2}(f, p, \psi)$ shows the anyone of the spaces $\bar{c}_{0}^{2}(f, p, \psi)$, $\bar{l}_{\infty}^{2}(f, p, \psi)$ and $\bar{l}_{p}^{2}(f, p, \psi)$. Let

$$
\bar{d}\left(\bar{B}_{k, l}, \overline{0}\right) \leq \bar{d}\left(\bar{A}_{k, l}, \overline{0}\right)
$$

holds for some $\left(\bar{A}_{k, l}\right) \in \bar{X}^{2}(f, p, \psi)$. Because the modulus function is increasing, we can clearly see by (3.8) that

$$
\begin{aligned}
& \lim _{k, l} \psi(k, l)\left[f\left(\bar{d}\left(\bar{B}_{k, l}, \overline{0}\right)\right)\right]^{p_{k, l}} \leq \lim _{k, l} \psi(k, l)\left[f\left(\bar{d}\left(\bar{A}_{k, l}, \overline{0}\right)\right)\right]^{p_{k, l}}, \\
& \sup _{k, l} \psi(k, l)\left[f\left(\bar{d}\left(\bar{B}_{k, l}, \overline{0}\right)\right)\right]^{p_{k, l}} \leq \sup _{k, l} \psi(k, l)\left[f\left(\bar{d}\left(\bar{A}_{k, l}, \overline{0}\right)\right)\right]^{p_{k, l},}, \\
& \sum_{k, l} \psi(k, l)\left[f\left(\bar{d}\left(\bar{B}_{k, l}, \overline{0}\right)\right)\right]^{p_{k, l}} \leq \sum_{k, l} \psi(k, l)\left[f\left(\bar{d}\left(\bar{A}_{k, l}, \overline{0}\right)\right)\right]^{p_{k, l}} .
\end{aligned}
$$

The above inequalities give that $\left(\bar{B}_{k, l}\right) \in \bar{X}^{2}(f, p, \psi)$.

Let $\inf _{k, l} p_{k, l}=h>0$. Then (a) If $\left(\bar{A}_{k, l}\right) \in \bar{c}^{2}$ then $\left(\bar{A}_{k, l}\right) \in \bar{c}^{2}(f, p, \psi)$, (b) If $\left(\bar{A}_{k, l}\right) \in \bar{c}^{2}(p, \psi)$ then $\left(\bar{A}_{k, l}\right) \in \bar{c}^{2}(f, p, \psi),(c)$ If $\beta=\lim _{t} \frac{f(t)}{t}>0$ then $\bar{c}^{2}(p, \psi)=\bar{c}^{2}(f, p, \psi)$. 
Proof. (a) Let $\left(\bar{A}_{k, l}\right) \in \bar{c}^{2}$. Then $\lim _{k, l} \bar{d}\left(\bar{A}_{k, l}, \bar{A}_{0}\right)=0$. Since $f$ is modulus function, then

$\lim _{k, l} f\left(\bar{d}\left(\bar{A}_{k, l}, \bar{A}_{0}\right)\right)=f\left[\lim _{k, l}\left(\bar{d}\left(\bar{A}_{k, l}, \bar{A}_{0}\right)\right)\right]=f(0)=0$.

Moreover $\inf _{k, l} p_{k, l}=h>0$, then $\lim _{k, l}\left[f\left(\bar{d}\left(\bar{A}_{k, l}, \bar{A}_{0}\right)\right)\right]^{h}=0$. Thus, for $0<\epsilon<1, \exists k_{0}$ such that for all $k, l>k_{0}\left[f\left(\bar{d}\left(\bar{A}_{k, l}, \bar{A}_{0}\right)\right)\right]^{h}<\epsilon<1$, as $p_{k, l} \geq h$ for all $k, l$,

$\left[\mathrm{f}\left(\bar{d}\left(\bar{A}_{k, l}, \bar{A}_{0}\right)\right)\right]^{p_{k, l}} \leq\left[f\left(\bar{d}\left(\bar{A}_{k, l}, \bar{A}_{0}\right)\right)\right]^{h}<\epsilon<1$,

then we have

$\lim _{k, l}\left[f\left(\bar{d}\left(\bar{A}_{k, l}, \bar{A}_{0}\right)\right)\right]^{p_{k, l}}=0$.

Since $\psi(k, l)$ is bounded, we can write

$\lim _{k, l} \psi(k, l)\left[f\left(\bar{d}\left(\bar{A}_{k, l}, \bar{A}_{0}\right)\right)\right]^{p_{k, l}}=0$.

Thus $\left(\bar{A}_{k, l}\right) \in \bar{c}^{2}(f, p, \psi)$.

(b) Suppose that $\left(\bar{A}_{k, l}\right) \in \bar{c}^{2}(p, \psi)$, then $\lim _{k, l} \psi(k, l)\left(\bar{d}\left(\bar{A}_{k, l}, \bar{A}_{0}\right)\right)^{p_{k, l}}=0$. Let $\epsilon>0$ and choose $\delta$ with $0<\delta<1$, such that $f(t)<\epsilon$ for $0 \leq t \leq \delta$. Now we define

$$
\mathrm{I}_{1}=\left\{k, l \in \mathbf{N}: \bar{d}\left(\bar{A}_{k, l}, \bar{A}_{0}\right) \leq \delta\right\}
$$

and

$$
\mathrm{I}_{2}=\left\{k, l \in \mathbf{N}: \bar{d}\left(\bar{A}_{k, l}, \bar{A}_{0}\right)>\delta\right\} .
$$

For $\bar{d}\left(\bar{A}_{k, l}, \bar{A}_{0}\right)>\delta$

$$
\bar{d}\left(\bar{A}_{k, l}, \bar{A}_{0}\right)<\bar{d}\left(\bar{A}_{k, l}, \bar{A}_{0}\right) \delta^{-1}<1+\left[\left|\bar{d}\left(\bar{A}_{k, l}, \bar{A}_{0}\right)\right|\right]
$$

where $k, l \in I_{2}$ and $[|t|]$ denotes the integer of $t$. From properties of modulus function, for $\bar{d}\left(\bar{A}_{k, l}, \bar{A}_{0}\right)>\delta$, we obtain

$\mathrm{f}\left(\bar{d}\left(\bar{A}_{k, l}, \bar{A}_{0}\right)\right)<1+\left[\left|\bar{d}\left(\bar{A}_{k, l}, \bar{A}_{0}\right)\right|\right] f(1) \leq 2 f(1) \bar{d}\left(\bar{A}_{k, l}, \bar{A}_{0}\right) \delta^{-1}$.

For $\bar{d}\left(\bar{A}_{k, l}, \bar{A}_{0}\right) \leq \delta, f\left(\bar{d}\left(\bar{A}_{k, l}, \bar{A}_{0}\right)\right)<\epsilon$, where $k, l \in I_{1}$. So $\psi(k, l)\left[f\left(\bar{d}\left(\bar{A}_{k, l}, \bar{A}_{0}\right)\right)\right]^{p_{k, l}}=\psi(k, l)\left[f\left(\bar{d}\left(\bar{A}_{k, l}, \bar{A}_{0}\right)\right)\right]^{p_{k, l}}$ \rfloor$\left._{k, l \in I_{1}}+\psi(k, l)\left[f\left(\bar{d}\left(\bar{A}_{k, l}, \bar{A}_{0}\right)\right)\right]^{p_{k, l}}\right\rfloor_{k, l \in I_{2}}$

$$
\leq \psi(k, l) \epsilon^{H}+\left[2 f(1) \delta^{-1}\right]^{H} \psi(k, l)\left[\bar{d}\left(\bar{A}_{k, l}, \bar{A}_{0}\right)\right]^{p_{k, l}} \rightarrow 0
$$

as $k, l \rightarrow \infty$

Then we obtain $\left(\bar{A}_{k, l}\right) \in \bar{c}^{2}(f, p, \psi)$.

(c) From $(b)$, we know that $\bar{c}^{2}(p, \psi) \subset \bar{c}^{2}(f, p, \psi)$. So we must show that $\bar{c}^{2}(f, p, \psi) \subset \bar{c}^{2}(p, \psi)$. For any modulus function, the existence of positive limit $\beta$ is given in Maddox [18]. Now, $\beta>0$ and let $\left(\bar{A}_{k, l}\right) \in \bar{c}^{2}(f, p, \psi)$. Since $\beta>0$ for every $t>0$, we write $f(t) \geq \beta$. From this inequality, we obtain that $\left(\bar{A}_{k, l}\right) \in \bar{c}^{2}(p, \psi)$. 
Let $f$ and $g$ be two modulus functions. Then

(a) $\bar{c}^{2}(f, p, \psi) \cap \bar{c}^{2}(g, p, \psi) \subset \bar{c}^{2}(f+g, p, \psi)$,

(b) Let $\psi_{1}, \psi_{2} \in M$ and $\psi_{1}(k, l) \leq \psi_{2}(k, l)$ for all $k, l \in \mathbf{N}$. Then $\bar{c}^{2}\left(f, p, \psi_{2}\right) \subset \bar{c}^{2}\left(f, p, \psi_{1}\right)$.

Proof. (a) Let $\left(\bar{A}_{k, l}\right) \in \bar{c}^{2}(f, p, \psi) \cap \bar{c}^{2}(g, p, \psi)$. From (2.1), we obtain

$\left[(\mathrm{f}+\mathrm{g})\left(\bar{d}\left(\bar{A}_{k, l}, \bar{A}_{0}\right)\right)\right]^{p_{k, l}}=\left[f\left(\bar{d}\left(\bar{A}_{k, l}, \bar{A}_{0}\right)\right)+g\left(\bar{d}\left(\bar{A}_{k, l}, \bar{A}_{0}\right)\right)\right]^{p_{k, l}}$

$\leq C\left[f\left(\bar{d}\left(\bar{A}_{k, l}, \bar{A}_{0}\right)\right)\right]^{p_{k, l}}+C\left[g\left(\bar{d}\left(\bar{A}_{k, l}, \bar{A}_{0}\right)\right)\right]^{p_{k, l}}$.

Since $\psi(k, l)$ is bounded, we can write

$\psi(k, l)\left[(f+g)\left(\bar{d}\left(\bar{A}_{k, l}, \bar{A}_{0}\right)\right)\right]^{p_{k, l}}$

$\leq C \psi(k, l)\left[f\left(\bar{d}\left(\bar{A}_{k, l}, \bar{A}_{0}\right)\right)\right]^{p_{k, l}}+C \psi(k, l)\left[g\left(\bar{d}\left(\bar{A}_{k, l}, \bar{A}_{0}\right)\right)\right]^{p_{k, l}}$.

Thus we have $\left(\bar{A}_{k, l}\right) \in \bar{c}^{2}(f+g, p, \psi)$.

(b) Let $\psi_{1}(k, l) \leq \psi_{2}(k, l)$ for all $k, l \in \mathbf{N}$.

Therefore

$\psi_{1}(k, l)\left[f\left(\bar{d}\left(\bar{A}_{k, l}, \bar{A}_{0}\right)\right)\right]^{p_{k, l}} \leq \psi_{2}(k, l)\left[f\left(\bar{d}\left(\bar{A}_{k, l}, \bar{A}_{0}\right)\right)\right]^{p_{k, l}}$.

This inequality shows that $\bar{c}^{2}\left(f, p, \psi_{2}\right) \subset \bar{c}^{2}\left(f, p, \psi_{1}\right)$.

Let $f$ be a modulus function, then $(a) \bar{l}_{\infty}^{2} \subset \bar{l}_{\infty}^{2}(f, p, \psi)$, (b) If $f$ is bounded then $\bar{l}_{\infty}^{2}(f, p, \psi)=\bar{\omega}^{2}$, where $\bar{\omega}^{2}$ is the set of all double sequence spaces of interval numbers.

Proof. (a) Suppose that $\left(\bar{A}_{k, l}\right) \in \bar{l}_{\infty}^{2}$. Then there exists a positive integer $M$ such that $\bar{d}\left(\bar{A}_{k, l}, \overline{0}\right) \leq M$. As $f$ is bounded then $f\left[\bar{d}\left(\bar{A}_{k, l}, \overline{0}\right)\right]$ is also bounded. Therefore

$\psi(k, l)\left[f\left(\bar{d}\left(\bar{A}_{k, l}, \overline{0}\right)\right)\right]^{p_{k, l}} \leq \psi(k, l)[M f(1)]^{p_{k, l}} \leq \psi(k, l)[M f(1)]^{H}<\infty$.

Hence $\left(\bar{A}_{k, l}\right) \in \bar{l}_{\infty}^{2}(f, p, \psi)$.

(b) Let $f$ be bounded. Then for any $\left(\bar{A}_{k, l}\right) \in \bar{\omega}^{2}$, $\psi(k, l)\left[f\left(\bar{d}\left(\bar{A}_{k, l}, \overline{0}\right)\right)\right]^{p_{k, l}} \leq \psi(k, l) L^{p_{k, l}} \leq \psi(k, l) L^{H}<\infty$.

Therefore $\bar{l}_{\infty}^{2}(f, p, \psi)=\bar{\omega}^{2}$.

\section{References}

[1] Chiao,K. P., Fundamental properties of interval vector max-norm, Tamsui Oxford Journal of Mathematics. 18, (2), pp. 219-233, (2002).

[2] Dwyer, P. S., Linear Computation, Wiley, New York, 1951. 
[3] Dwyer, P. S., Error of matrix computation, simultaneous equations and eigenvalues, National Bureu of Standarts, Applied Mathematics Series. 29, pp. 49-58, (1953).

[4] Esi, A., Strongly almost $\lambda$-convergence of interval numbers, Scientia Magna, 7 (2), pp. 117-122, (2011).

[5] Esi, A., Lacunary sequence spaces of interval numbers, Thai Journal of Mathematics, 10 (2), pp. 445-451, (2012).

[6] Esi, A., $\lambda$ sequence spaces of interval numbers, Appl. Math. Inf. Sci, 8, (3), pp. 1099-1102, (2014).

[7] Esi, A., A new class of interval numbers, Journal of QafQaz University, Mathematics and Computer Sciences, pp. 98-102, (2012).

[8] Esi, A., Double lacunary sequence spaces of double sequence of interval numbers, Proyecciones Journal of Mathematics. 31, (1), pp. 297-306, (2012).

[9] Esi, A. and Yasemin, S., Some spaces of sequences of interval numbers defined by a modulus function, Global Journal of Mathematical Analysis, 2, (1), pp. 11-16, (2014).

[10] Esi, A., A new class of double interval numbers, Scientia Magna, 4 (7), pp. 23-28, (2011).

[11] Esi, A., Statistical and lacunary statistical convergence of interval numbers in topological groups, Acta Scientarium. Techno., 36 (3), pp. 491- 495, (2014).

[12] Esi, A. and Hazarika, B., Some double sequence spaces of interval numbers defined by Orlicz function, Journal of the Egyptian Mathematical Society, 22, (3), pp. 424-427, (2014).

[13] Esi, A and Esi, A., Asymptotically lacunary statistically equivalent sequences of interval numbers, Int. J. Math. Appl., 1 (1), pp. 43-48, (2013).

[14] Esi, A. and Braha, N., On asymptotically $\lambda$-statistical equivalent sequences of interval numbers, Acta Scientarium. Techno., 35 (3), pp. 515-520, (2013). 
[15] Esi, A., Strongly $\lambda$-Summable Sequences of interval numbers, International Journal of Science, Environment and Technology, 5 (6), pp. 4643-4648, (2016).

[16] Esi, A. and Catalbas, N., Some sequence spaces of interval numbers defined by Orlicz functions, Proceedings of the Jangjeon Mathematical Society, 20 (1), pp. 35-41, (2017).

[17] Fischer, P. C., Automatic propagated and round-off error analysis, paper presented at the 13th National Meeting of the Association of Computing Machinary, June (1958).

[18] Maddox, I. J., Spaces of strongly summable sequences, Quart. J. Math., Oxford Ser. 18, (2), pp. 345-355, (1967).

[19] Markov, S., Quasilinear spaces and their relation to vector spaces, Electronic Journal on Mathematics of Computation, 2, (1), (2005).

[20] Moore, R. E., Automatic Error Analysis in Digital Computation, LSMD-48421, Lockheed Missiles and Space Company, (1959).

[21] Moore, R. E. and Yang, C. T., Theory of an Interval Algebra and Its Application to Numeric Analysis, RAAG Memories II, Gaukutsu Bunken Fukeyu-kai, Tokyo, (1958).

[22] Moore, R. E. and Yang, C. T., Interval Analysis I, LMSD-285875, Lockheed Missiles and Space Company, (1962).

[23] Pringsheim, A., Zur Theorie der zweifach unendlichen Zahlenfolgen, Math. Ann. 53, pp. 289-321, (1900).

[24] Şengönül, M. and Eryılmaz, A., On the sequence spaces of interval numbers, Thai Journal of Mathematics. 8, (3), pp. 503-510, (2010).

\author{
Sibel Yasemin Gölbol \\ Mersin University \\ Department of Mathematics \\ 33343 Yenisehir/Mersin, \\ Turkey \\ e-mail : sibelyasemin@mersin.edu.tr
}




\section{Ayhan Esi}

Adiyaman University

Department of Mathematics

02040 Adıyaman,

Turkey

e-mail : aesi23@hotmail.com

and

Uğur Değer

Mersin University

Department of Mathematics

33343 Yenisehir/Mersin,

Turkey

e-mail : udeger@mersin.edu.tr 